\title{
Neumotórax espontáneo y neumomediastino en neumonía por SARS-CoV-2: serie de casos
}

\section{Spontaneous pneumothorax and pneumomediastinum in SARS-CoV-2 pneumonia: case series}

\author{
Marysabel Cogollo-González,* Marlyn Zamora-Posada,* Catalina Sanmiguel-Reyes, ${ }^{*}$ \\ Juan Pablo Rodríguez-Gallego, ${ }^{\ddagger}$ Rafael Enrique Conde-Camacho ${ }^{\ddagger}$
}

*Universidad de la Sabana, Cundinamarca, Colombia; ”Fundación Cardioinfantil-Instituto de Cardiología y Fundación Neumológica Colombiana, Bogotá, Colombia.

\begin{abstract}
RESUMEN. Introducción: El neumotórax y el neumomediastino asociados a la COVID-19 son complicaciones raras, con una incidencia entre 0.56 y $2 \%$. Pueden desarrollarse en diferentes fases de la enfermedad y en ausencia de factores de riesgo. Objetivo: Describir dos casos de neumotórax y un caso de neumomediastino asociados con neumonía por COVID-19. Material y métodos: Serie de casos retrospectiva. Resultados: Dentro de las características demográficas se destacan la edad entre 50 y 70 años, ausencia de comorbilidades respiratorias o tabaquismo. La media de tiempo entre el inicio de síntomas hasta la aparición del evento fue de 14.6 días. Se presentó un único caso con neumotórax bilateral y neumomediastino de manera simultánea. El tratamiento consistió en manejo conservador para el caso de neumomediastino, toracostomía cerrada para los casos de neumotórax, uno de ellos con requerimiento de pleurectomía con decorticación. La media de estancia hospitalaria fue de 31.3 días, con una evolución clínica satisfactoria de la totalidad de los pacientes. Conclusiones: El neumotórax y el neumomediastino son complicaciones potencialmente fatales de la COVID-19. Pueden presentarse en ausencia de enfermedad respiratoria, tabaquismo y ventilación mecánica. Deben sospecharse en todo paciente con deterioro respiratorio en el curso de la enfermedad.
\end{abstract}

Palabras clave: Complicaciones, COVID-19, enfisema mediastinal, neumotórax, lesión pulmonar.

\section{INTRODUCCIÓN}

El neumotórax se define como la acumulación de aire en el espacio pleural que puede ocurrir sin eventos desenca-

\section{Correspondencia:}

Dra. Marysabel Cogollo-González

Universidad de la Sabana, Chía-Cundinamarca, Colombia.

Correo electrónico: marysabelcogo@unisabana.edu.co

Recibido: 24-IV-2021; aceptado: 18-V-2021.

Citar como: Cogollo-González M, Zamora-Posada M, Sanmiguel-Reyes C, Rodríguez-Gallego JP, Conde-Camacho RE. Neumotórax espontáneo y neumomediastino en neumonía por SARS-CoV-2: serie de casos. Neumol Cir Torax. 2021; 80 (3): 222-229. https://dx.doi.org/10.35366/102485
ABSTRACT. Introduction: Pneumothorax and pneumomediastinum associated with COVID-19 are rare complications, with an estimated incidence of 0.56 to $2 \%$ of cases. They can occur in different phases of the disease and in absence of risk factors. Objective: To describe two pneumothorax cases and other with pneumomediastinum associated with COVID-19 pneumonia. Material and methods: Retrospective case series. Results: Within the demographic characteristics, the age between 50 to 70 years old stands out, no respiratory comorbidities or smoking. The mean time between the onset of symptoms and the event was 14.6 days. There was a single case with concomitant bilateral pneumothorax with pneumomediastinum. Treatment consisted of conservative management for the pneumomediastinum. Both pneumothorax cases performed closed thoracostomy, one of them required pleurectomy with decortication due to non-re-expansion of the compromised lung. The mean hospital stay was 31.3 days. All patients had a satisfactory clinical evolution. Conclusions: Pneumothorax and pneumomediastinum are potentially fatal complications of COVID-19. It can occur in the absence of respiratory disease, previous smoking or mechanical ventilation. It should be suspected in all patients who present respiratory deterioration in the course of the disease.

Keywords: Complications, COVID-19, mediastinal emphysema, pneumothorax, lung injury.

denantes en una persona sin enfermedad pulmonar, denominado neumotórax espontáneo primario, o surgir como una complicación de una enfermedad pulmonar o trauma subyacente conocido como neumotórax secundario. ${ }^{1}$

En diciembre de 2019 se describieron varios casos de neumonía de etiología desconocida en Wuhan (China), ${ }^{2,3}$ cuyo desarrollo posteriormente fue explicado por la identificación de un nuevo coronavirus que se denominó SARS-CoV-2, y a la enfermedad desencadenada por el virus, COVID-19. ${ }^{4}$ Desde entonces, se han asociado diferentes complicaciones, que incluyen el síndrome de dificultad respiratoria aguda (SDRA), las coagulopatías, la insuficiencia renal y las alteraciones cardíacas y neurológicas. ${ }^{5}$

Respecto a las enfermedades por coronavirus registradas previamente, el neumotórax espontáneo ha sido 
descrito como una complicación del SDRA causado por el SARS-CoV-1, con una incidencia de $1.7 \%$ en pacientes hospitalizados, ${ }^{6}$ y su aparición constituye un factor de mal pronóstico en el síndrome respiratorio del Medio Oriente (MERS). ${ }^{7}$ El neumotórax asociado con la COVID-19 tiene una incidencia entre 0.56 y $2 \%,{ }^{8-12}$ y dentro de los presuntos mecanismos fisiopatológicos se plantean los cambios estructurales en el parénquima pulmonar producidos por el virus que conducen a daño alveolar difuso (DAD), descamación evidente de neumocitos y formación de membranas hialinas. ${ }^{13,14}$ Lo anterior propicia el desarrollo de neumatoceles y predispone a la aparición del neumotórax en los diferentes estadíos de la enfermedad..$^{15}$ Entre otros factores de riesgo se encuentran los que aumentan la presión intratorácica como el uso de la ventilación mecánica (VM) y la tos prolongada. ${ }^{8,16}$

Por otro lado, el neumomediastino puede deberse a una fuga de aire a través del espacio intersticial secundario al aumento de la presión. ${ }^{17}$ El DAD y la rotura alveolar conllevan a enfisema intersticial y extensión del aire a lo largo de la vaina broncoalveolar hasta el mediastino. ${ }^{4}$ Dentro de los eventos que contribuyen a su desarrollo, se describen el tabaquismo, el consumo de drogas ilícitas, la enfermedad pulmonar intersticial, las infecciones pulmonares y la realización de maniobras de Valsalva vigorosas., ${ }^{4,18}$

Los casos de neumonía por COVID-19 que cursan con neumotórax bilateral y neumomediastino durante su evolución son raros, y al momento existen pocos reportes en la literatura. ${ }^{4,19}$ Nuestro objetivo es describir una serie de casos de neumotórax espontáneo y neumomediastino en el contexto de neumonía por COVID-19.

\section{REPORTE DE CASOS}

Caso 1. Masculino de 59 años, sin comorbilidades o tabaquismo previo. Consultó por mialgias, tos seca, anosmia y fiebre. Los signos vitales en la primera consulta se encontraban normales, con reporte de la reacción en cadena de la polimerasa con transcripción reversa (RT-PCR) para SARSCoV-2 positiva. Se consideró infección leve por COVID-19 y se indicó aislamiento en casa. Al noveno día de síntomas, reingresó a la institución por disnea súbita y dolor pleurítico derecho. Al examen físico con hipertensión, taquicardia, taquipnea y saturación arterial de oxígeno $\left(\mathrm{SaO}_{2}\right)$ en $86 \%$. Los paraclínicos de ingreso demostraban linfopenia (leucocitos $7,910 / \mathrm{mm}^{3}$, linfocitos $950 / \mathrm{mm}^{3}$ [12\%]) y elevación de los marcadores de severidad: proteína $C$ reactiva 7.11 $\mathrm{mg} / \mathrm{dL}(<5 \mathrm{mg} / \mathrm{dL})$, dímero D (DD) $840 \mathrm{ng} / \mathrm{mL}(<500 \mathrm{ng} /$ $\mathrm{mL})$, lactato deshidrogenasa $(\mathrm{LDH}) 441 \mathrm{U} / \mathrm{L}(85-227 \mathrm{U} / \mathrm{L})$ y ferritina $717 \mathrm{ng} / \mathrm{mL}$ (12 a $200 \mathrm{ng} / \mathrm{mL}$ ).

La radiografía de tórax demostró un neumotórax derecho con colapso de 50\% del hemitórax ipsilateral (Figura $1 \mathrm{~A})$, que requirió manejo con toracostomía cerrada derecha y expansión completa pulmonar en los controles radiográficos. La angiotomografía (angio-TC) de tórax descartó tromboembolismo pulmonar (TEP) y evidenció opacidades en vidrio esmerilado (OVE) bilaterales, de distribución periférica, subpleural, con engrosamiento septal interlobulillar y enfisema subcutáneo derecho (Figuras 1B y C). Se consideró neumonía por COVID-19, y su manejo consistió en dexametasona intravenosa (IV) de acuerdo al ensayo RECOVERY, ${ }^{20}$ oxígeno $\left(\mathrm{FiO}_{2} 32 \%\right)$ y analgesia. A las 72 horas se retiró el tubo de tórax. Los laboratorios de control mostraron disminución de los marcadores de severidad, con egreso a los siete días de estancia hospitalaria. En el seguimiento a los tres meses (Tabla 1), la puntuación de la escala de disnea modificada del Medical Research Council (mMRC) fue de cero, sin alteraciones en las pruebas de función pulmonar, con OVE residuales en la tomografía control.

Caso 2. Masculino de 50 años, con antecedentes de hipertensión arterial (HTA) y sobrepeso, sin patologías respiratorias, tabaquismo o consumo previo de drogas ilícitas. Presentó infección moderada por SARS-CoV-2, con ingreso hospitalario para manejo con oxígeno por cánula nasal $\left(\mathrm{FiO}_{2} 32 \%\right.$ ) y esteroide IV según el ensayo RECOVERY, ${ }^{20} \mathrm{Con}$ posterior egreso. En el día 20 desde el inicio de síntomas
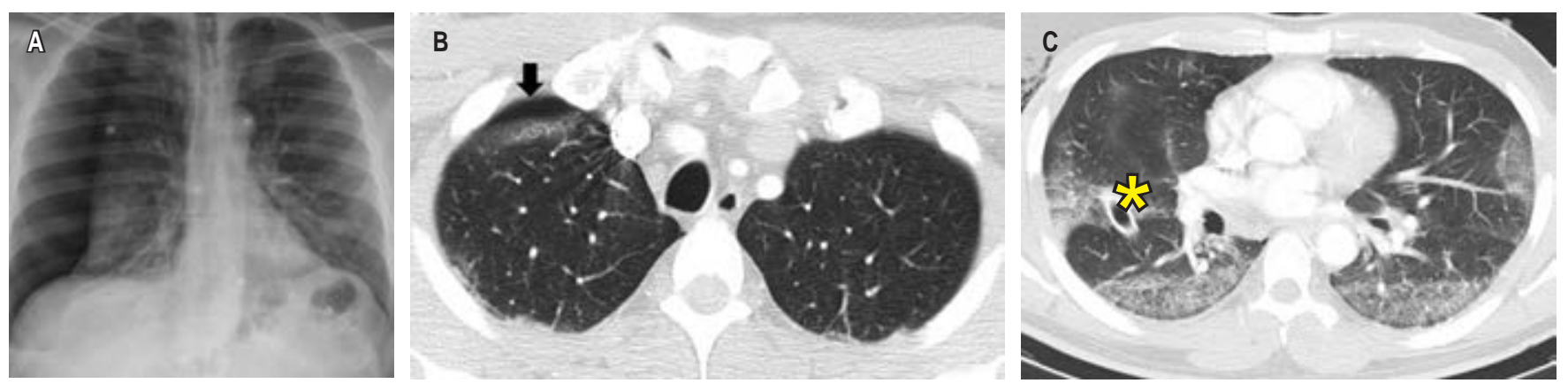

Figura 1: Radiografía de tórax, proyección posteroanterior. A) Neumotórax derecho. B, C) Angio-TC de tórax, ventana para pulmón, plano axial. Se observa cámara de neumotórax (flecha negra), vidrio esmerilado de predominio en lóbulos inferiores, tubo de tórax (asterisco) y enfisema subcutáneo derecho. 
Tabla 1: Seguimiento de los pacientes a los tres meses.

\begin{tabular}{|c|c|c|c|c|c|c|c|}
\hline Caso & $\begin{array}{c}\text { Disnea } \\
\text { mMRC* }\end{array}$ & Oxígeno & Espirometría & $\begin{array}{c}\text { DLCO ajustada } \\
\text { a la altura }\end{array}$ & $\begin{array}{c}\text { TC6M (\% de } \\
\text { lo esperado) }\end{array}$ & $\begin{array}{c}\text { Rehabilitación } \\
\text { pulmonar }\end{array}$ & TCAR pulmonar control \\
\hline 1 & 0 & No & Normal & Normal $(90 \%)$ & $\begin{array}{c}\text { No } \\
\Delta \mathrm{SaO}_{2}:-2 \%\end{array}$ & $\begin{array}{c}\text { Algunas OVE subpleurales } \\
\text { residuales bilaterales y } \\
\text { basales }\end{array}$ \\
\hline 2 & 1 & $\begin{array}{c}\text { Sí } \\
(1 \mathrm{~L} / \mathrm{min})\end{array}$ & $\mathrm{SD}$ & $\mathrm{SD}$ & $\begin{array}{c}615 \mathrm{~m}(92) \\
\Delta \mathrm{SaO}_{2}:-9 \%\end{array}$ & $\begin{array}{c}\text { Sí } \\
\text { nesión cicatrizal en LSI por } \\
\text { bilaterales }\end{array}$ \\
\hline 3 & 2 & No & $\begin{array}{c}\text { Sugestiva de } \\
\text { restricción }\end{array}$ & $\begin{array}{c}\text { Moderadamente } \\
\text { disminuida } \\
(55 \%)\end{array}$ & $\begin{array}{c}\Delta \mathrm{SaO}_{2}:-7 \% \\
\mathrm{~m}(82)\end{array}$ & $\begin{array}{c}\text { Sí } \\
\text { OVE residuales y bandas } \\
\text { parenquimatosas bilaterales } \\
\text { y basales. No neumome- } \\
\text { diastino }\end{array}$ \\
\hline
\end{tabular}

*Escala de disnea modificada del Medical Research Council (mMRC).

DLCO = capacidad de difusión de monóxido de carbono; TC6M = test de caminata de 6 minutos; TCAR = tomografía de alta resolución; $\triangle \mathrm{SaO} 2$ = delta de saturación arterial de oxígeno; LSI = lóbulo superior izquierdo; OVE = opacidades en vidrio esmerilado; SD = sin datos.
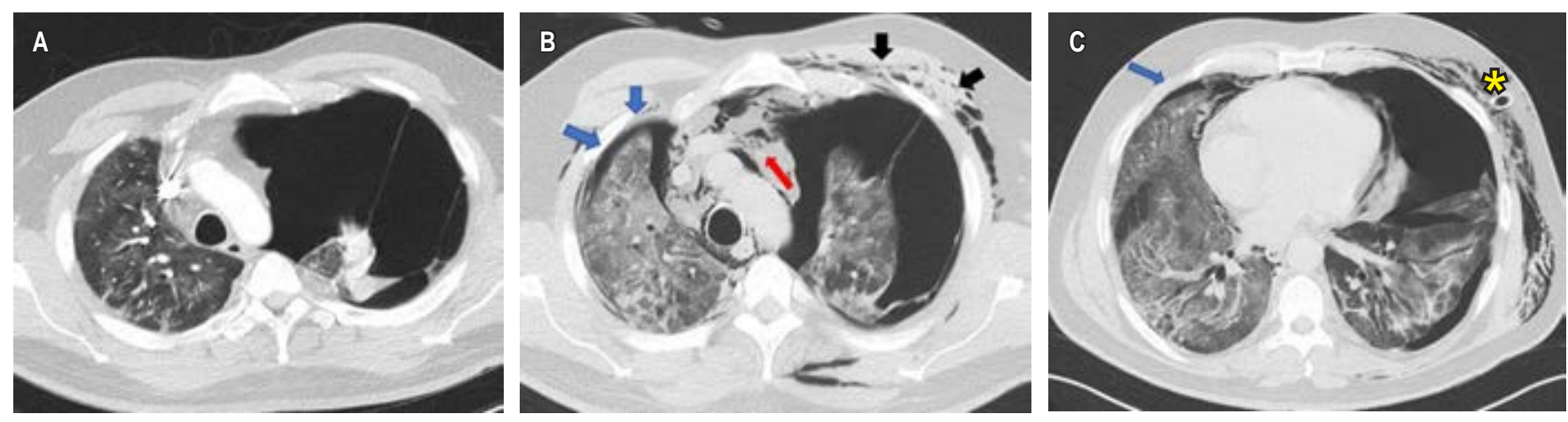

Figura 2: Angio-TC, ventana para pulmón, corte axial: A) Neumotórax izquierdo y opacidades en vidrio esmerilado. B, C) TC (tomografía computarizada) de tórax control, cortes axiales con neumotórax derecho (flechas azules), neumomediastino (flecha roja), persistencia del neumotórax izquierdo (flecha negra), enfisema subcutáneo y patrón de crazy paving. Se observa además tubo de tórax (asterisco).

desarrolló disnea súbita y dolor retroesternal izquierdo, por lo que reconsultó a urgencias. Los signos vitales de ingreso mostraron taquicardia (120 latidos/minuto), taquipnea (26 respiraciones/minuto), y desaturación $\left(\mathrm{SaO}_{2} 70 \%\right)$. Paraclínicos con leucocitosis $\left(12,400 / \mathrm{mm}^{3}\right)$, neutrofilia $(10,500 /$ $\left.\mathrm{mm}^{3}[84.4 \%]\right)$, linfopenia $\left(1,000 / \mathrm{mm}^{3}[8.08 \%]\right)$ y elevación de los marcadores de severidad: DD 990 ng/mL, LDH 408 $\mathrm{U} / \mathrm{L}$ y ferritina $380 \mathrm{ng} / \mathrm{mL}$.

La angio-TC de tórax fue negativa para TEP, con evidencia de OVE del lado derecho y neumotórax contralateral que colapsaba $90 \%$ del pulmón (Figura 2A), por lo que se realizó una toracostomía cerrada izquierda de urgencia, sin lograrse la expansión pulmonar. Al tercer día de estancia hospitalaria, presentó deterioro de la oxigenación que requirió inicio de suplencia de oxígeno por cánula de alto flujo e ingresó a la unidad de cuidados intensivos (UCI) sin requerimiento de VM. La TC de alta resolución control (Figuras $2 B$ y C) demostró OVE, áreas de crazy paving de predomino derecho, neumotórax apical derecho pequeño $(<10 \%)$, neumomediastino y enfisema de los tejidos blandos del hemitórax izquierdo. Fue llevado a una segunda toracostomía cerrada a nivel apical izquierdo por cirugía de tórax, con persistencia del colapso pulmonar ipsilateral, por lo que se realizó decorticación y pleurectomía parietal por toracoscopia, con expansión satisfactoria. La patología reportó pleuritis crónica (Figura 3). Se indicó manejo conservador del neumotórax derecho, con expansión pulmonar en imágenes posteriores.

El reporte de imágenes previas en tórax aportado por el paciente no evidenciaba los hallazgos en el parénquima pul- 
monar durante esta hospitalización. Los estudios de autoinmunidad fueron negativos. Se consideró cuadro secundario a las complicaciones asociadas con neumonía severa por COVID-19. Por evolución favorable fue egresado a los 42 días de estancia hospitalaria. En la valoración a los 90 días (Tabla 1) se encontraba en rehabilitación pulmonar, continuaba con oxígeno a $1 \mathrm{~L} / \mathrm{min}$, refirió mejoría de la disnea, con una distancia recorrida por el test de caminata de seis minutos (TC6M) de 615 metros y un delta de $\mathrm{SaO}_{2}$ mayor de tres puntos. La TC control mostró lesión cicatricial en el lóbulo superior izquierdo por neumotórax y escasas OVE.

Caso 3. Masculino de 64 años, sin antecedentes relevantes ni tabaquismo. Ingreso hospitalario al día 11 de inicio de síntomas dados por disnea asociada a desaturación a medio ambiente $\left(\mathrm{SaO}_{2} 85 \%\right)$, con confirmación molecular de infección por SARS-CoV-2 y radiografía de tórax con OVE difusas. El tratamiento inicial consistió en oxígeno ( $\mathrm{FiO}, 32 \%)$, antipiréticos y dexametasona IV según el ensayo RECOVERY. ${ }^{20} \mathrm{Al}$ día cuarto de estancia (día 15 de síntomas) presentó tos persistente, mayor deterioro del patrón respiratorio e hipoxemia severa sin mejoría con máscara de no reinhalación ( $\mathrm{FiO}_{2} 90 \%$ ), ni pronación, además presencia de crepitaciones cervicales izquierdas. Paraclínicos con leucocitosis $(16,600 \mathrm{cel} / \mu \mathrm{L})$, linfopenia (300 cel $/ \mathrm{cel} / \mu \mathrm{L})$, elevación de la LDH (524 U/L), hiperferritinemia $(4,519 \mathrm{ng} / \mathrm{mL})$ y DD en $630 \mathrm{ng} / \mathrm{mL}$. Requirió intubación orotraqueal y traslado a la UCI. Se consideró descartar complicaciones de la COVID-19, se realizó una angio-TC de tórax que mostró TEP agudo y neumomediastino con enfisema cervical izquierdo (Figura 4). Se indicó anticoagulación con heparina de bajo peso molecular (1 mg por kg cada 12 horas), antibióticos y VM. Se realizaron otros estudios como fibrobroncoscopia y endoscopia de vías digestivas altas sin evidenciar hallazgos anormales. Se consideró neumomediastino espontáneo asociado a neumonía severa por SARS-CoV-2. Cirugía de tórax optó por manejo conservador, con una evolución hacia la mejoría y egreso al día 40. A los tres meses se realizaron pruebas de función pulmonar con espirometría sugestiva de restricción y capacidad de monóxido de car- bono (DLCO), moderadamente disminuida (Tabla 1). En la actualidad continúa en sesiones de rehabilitación pulmonar con mejoría de la disnea.

\section{DISCUSIÓN}

Los casos presentados describen pacientes con neumonía por COVID-19 sin antecedentes de enfermedades pulmonares, traumatismo torácico, VM o exposición significativa al tabaco, que desarrollaron neumotórax y/o neumomediastino espontáneo.

Según la serie de casos reportada por Martinelli et al. que evaluó 71 pacientes con infección por SARS-CoV-2, de los cuales 60 tuvieron neumotórax y 11 sólo neumomediastino, dentro de las características demográficas y clínicas más frecuentes se encuentran el sexo masculino, la edad de presentación entre 50 y 80 años, el compromiso unilateral del neumotórax (principalmente del lado derecho), la ausencia de hábito tabáquico y pocas comorbilidades médicas, siendo la más común la HTA. ${ }^{9}$ En relación con la fase de la enfermedad, se puede presentar al inicio, durante la hospitalización o de forma tardía. ${ }^{8,9}$ Wang y colaboradores reportaron un caso de infección por COVID-19 con neumotórax y neumomediastino de aparición en el día 20 de inicio de síntomas, de manera similar a nuestro segundo caso descrito. ${ }^{21}$

Los hallazgos imagenológicos distintivos de la neumonía por COVID-19 son OVE periféricas, subpleurales y bilaterales, que posteriormente progresan a consolidaciones, opacidades lineales, patrón de crazy paving y signo del «halo inverso». Lo anterior representa la correlación de los cambios tomográficos con la fisiopatología subyacente al proceso de la enfermedad a medida que se organiza. ${ }^{22}$ Diversos estudios han demostrado que en algunos pacientes con infección por COVID-19 hay progresión radiológica de áreas de consolidación a espacios aéreos anormales en la TC. ${ }^{16,23}$ Estos cambios quísticos redondos en la tomografía pueden estar asociados con el proceso de reabsorción de la consolidación, lo que podría explicarse
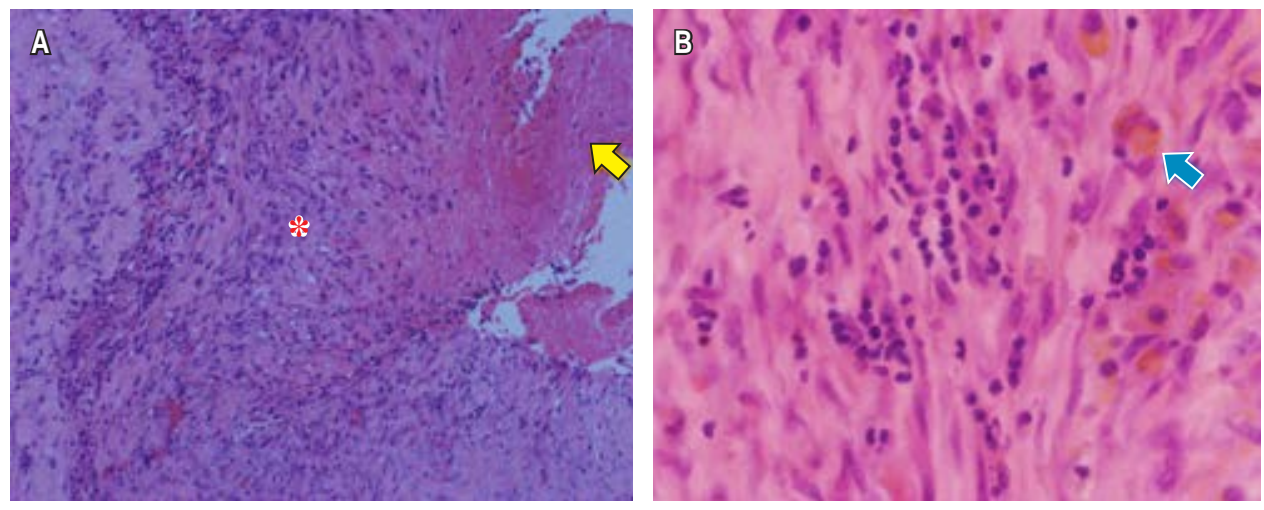

Figura 3:

Biopsia de pleura. A) Tinción con hematoxilina y eosina $(10 x)$, se observa fibrina (flecha), con fibrosis e infiltrado inflamatorio crónico (asterisco). B) Se observa infiltrado linfoplasmocitario y presencia de hemosiderófagos (flecha azul). Hematoxilina y eosina (40x). 
Tabla 2: Resumen de casos de pacientes infectados por SARS-CoV-2 con neumomediastino y neumotórax.

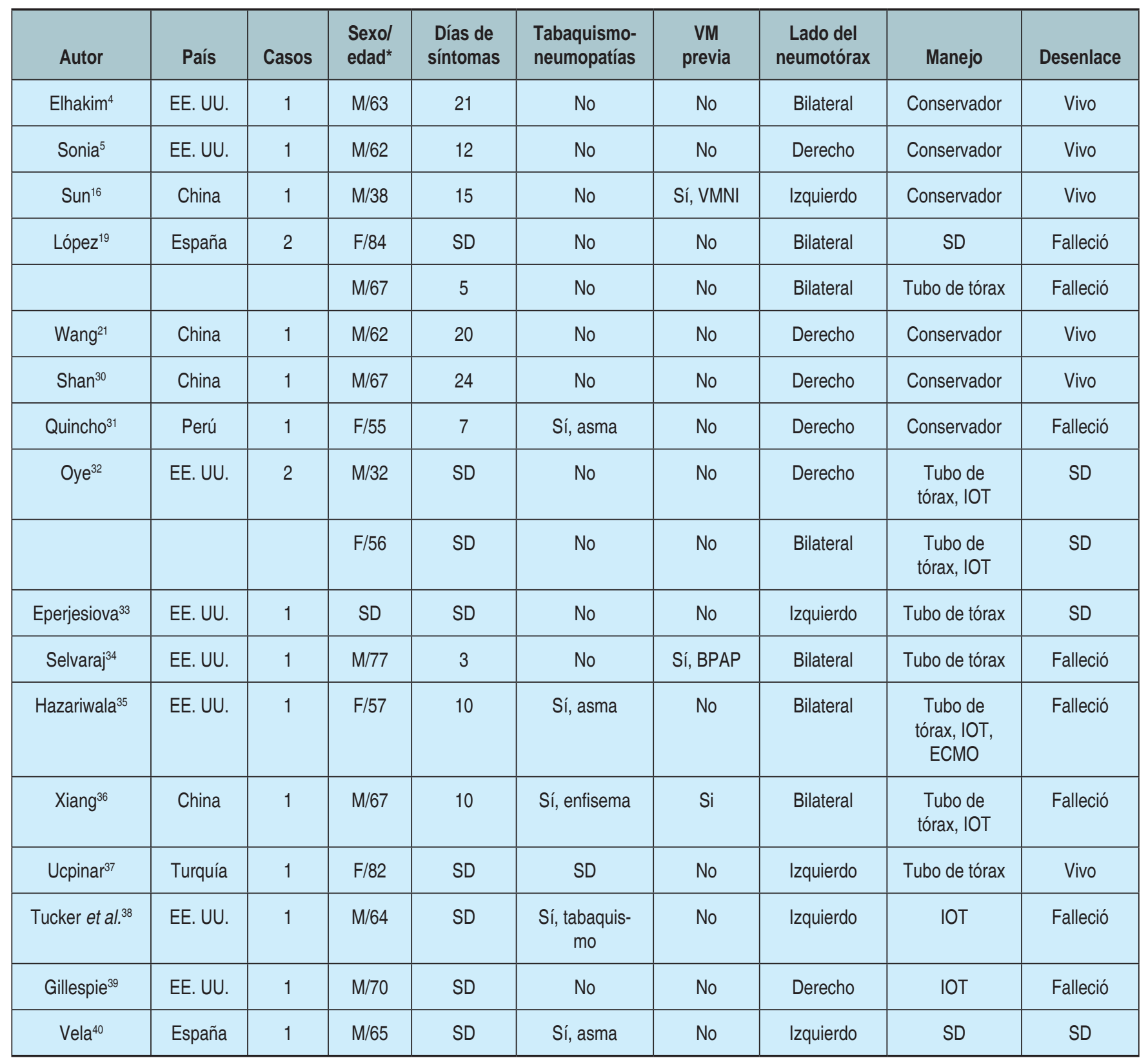

* En años. EE. UU. = Estados Unidos; M = masculino; F = femenino; SD = sin datos; VM = ventilación mecánica; VMNI = ventilación mecánica no invasiva; IOT = intubación orotraqueal; $\mathrm{BPAP}=$ presión positiva de dos niveles en las vías respiratorias; $\mathrm{ECMO}=$ sistema de oxigenación por membrana extracorpórea.

por el daño causado a las paredes alveolares secundario a la infección, que conduce a neumatoceles e incrementa el riesgo de desarrollar neumotórax. ${ }^{24}$ Nuestros casos mostraron hallazgos radiológicos típicos de infección por COVID-19 con presencia de crazy paving en el segundo caso, que sugieren la relación temporal de los hallazgos imagenológicos y una evolución probablemente más tardía de la enfermedad.
La patología del segundo caso fue similar a lo documentado por Chang et al., quienes encontraron en algunos pacientes pleuritis crónica en muestras histopatológicas de pacientes llevados a cirugía torácica por complicaciones por COVID-19. ${ }^{25}$

La Tabla 2 resume las características clínicas y demográficas de algunos informes de casos de infección por SARS-CoV-2, donde se presentaron de manera simultánea 
neumotórax y neumomediastino, asociación infrecuente en la literatura.

El manejo de esta condición dependerá de la extensión del neumotórax y del estado general del paciente. En neumotórax parciales con estabilidad clínica se recomienda el manejo conservador y la vigilancia radiológica para descartar la progresión de esta entidad. En caso contrario, se indica la evacuación del aire mediante aspiración o drenaje. ${ }^{26}$ Los procedimientos en pleura pueden ser posibles generadores de aerosoles, por lo que los trabajadores de la salud deben hacer uso de todo el equipo de protección personal (EPP) y gestionar medidas para minimizar la propagación del virus durante la realización de estas intervenciones. ${ }^{27}$ En el estudio de Chang, ningún proveedor de atención médica fue positivo para COVID-19 después de las cirugías torácicas. ${ }^{25}$ En nuestros casos se utilizaron los EPP y se optó por manejo conservador y toracostomía cerrada como primera intervención quirúrgica. Ante la falta de respuesta al manejo inicial, se realizó decorticación y pleurectomía con todas las medidas de bioseguridad y en quirófano con presión negativa.

La persistencia de fuga de aire (de tres a cinco días) o la imposibilidad para lograr la expansión pulmonar son indicaciones de cirugía. ${ }^{26}$ La técnica quirúrgica se basa en la identificación y resección de áreas diana o ampollosas y una técnica de pleurodesis asociada, ya sea abrasión mecánica de la pleura parietal, pleurectomía química o apical. ${ }^{26,28}$ En la serie de casos por Chang, se describieron 13 pacientes con infección por COVID-19 sometidos a cirugías torácicas, dos por neumotórax y tres por neumatoceles. ${ }^{25}$ Aiolfi reportó dos pacientes con COVID-19 que fueron tratados exitosamente con pleurectomía por neumotórax persistente. ${ }^{29}$

El neumomediastino, en la mayoría de los casos, tiene un curso natural benigno con manejo conservador, que consiste en un estrecho seguimiento, reposo en cama, analgesia y oxigenoterapia. ${ }^{4}$ En nuestro caso, la evolución fue satisfactoria.

Nuestros pacientes tuvieron un seguimiento a los tres meses con mejoría de la sintomatología, en quienes necesitaron $\mathrm{UCl}$ se prescribió rehabilitación pulmonar y en el tercer caso se documentó alteración de la DLCO y espirometría sugestiva de restricción. Aunque hubo una buena distancia recorrida en el TC6M, en dos casos se observó un delta de $\mathrm{SaO}_{2}$ mayor de tres puntos durante la prueba. Los hallazgos imagenológicos mostraron mejoría y disminución de las lesiones en el tiempo.

Los estudios reportados a la fecha han sido de carácter observacional, lo cual impide establecer relaciones de causalidad entre el neumotórax, el neumomediastino y la infección por SARS-CoV-2. La serie de Martinelli et al. no evidenció que el neumotórax y el neumomediastino incrementaran la mortalidad a 28 días en los pacientes con COVID-19; sin embargo, la presencia de acidosis y la edad avanzada en ese contexto se relacionaron con una baja supervivencia. ${ }^{9}$ Recientemente, Miró et al. informaron que los pacientes con COVID-19 y neumotórax espontáneo tuvieron mayor riesgo de muerte intrahospitalaria en comparación con los dos grupos control (pacientes con COVID-19 sin neumotórax y aquéllos con neumotórax y sin infección por SARS-CoV-2). No obstante, los autores señalan que el tamaño muestral pequeño impide el ajuste de las variables vinculadas a la gravedad de la COVID-19. ${ }^{12}$ Por lo tanto, se requieren más estudios para determinar el pronóstico de estos pacientes.

La caracterización de los pacientes con estas complicaciones ofrece una orientación inicial que permite sugerir qué pacientes requieren un seguimiento más estricto, dado el riesgo de deterioro clínico y mortalidad. Esta serie de casos evidencia que su aparición no se limita a los pacientes críticamente enfermos y no sólo está determinada por la VM, sino que tal vez se explica por la fisiopatología de la enfermedad.

\section{CONCLUSIONES}

La neumonía por SARS-CoV-2 puede presentar complicaciones en el curso de la enfermedad. Las principales causas son el TEP y la sobreinfección bacteriana; sin embargo, se deben considerar otros eventos infrecuentes como el neumotórax y/o neumomediastino, que pueden ser potencialmente fatales y ocurrir en pacientes sin antecedentes de traumatismo, tabaquismo o VM. Este grupo de pacientes en riesgo reque-
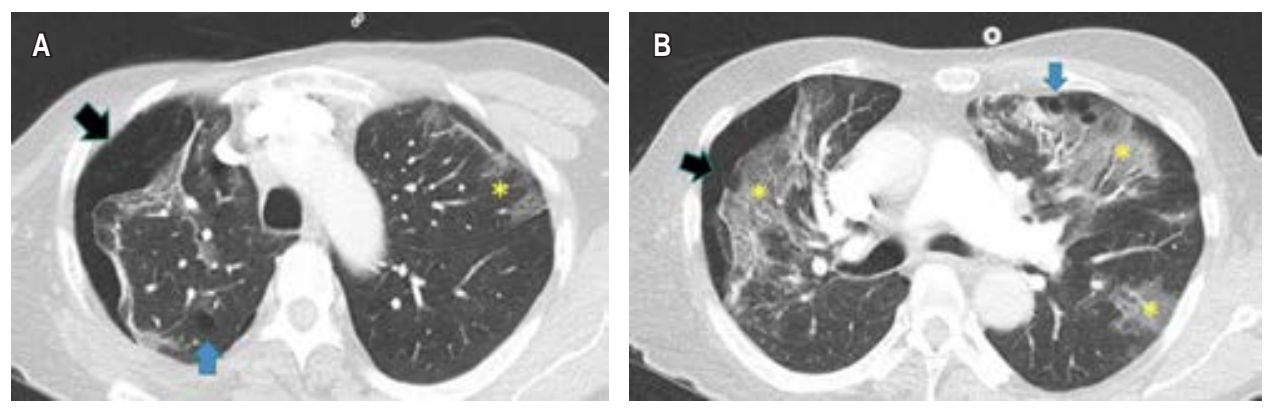

Figura 4:

Angio-TC, ventana para pulmón corte axial (A) y coronal (B). Se observan opacidades en vidrio esmerilado, neumomediastino (flechas) y enfisema de los tejidos blandos de la región cervicotorácica izquierda (asterisco). 
riría un seguimiento más estricto ante el riesgo de deterioro clínico y mortalidad. El mecanismo presuntivo es el DAD con posterior ruptura alveolar y formación de neumatoceles. En su manejo son importantes las medidas de protección por parte del personal de la salud para minimizar la aerolización.

\section{Agradecimientos}

A los pacientes y todo el personal de la Fundación Cardioinfantil-Instituto de Cardiología, Bogotá, Colombia.

\section{REFERENCIAS}

1. McCool FD. Diseases of the diaphragm CW, pleura and mediastinum. In: Goldman L, Schafer Al, editors. Goldman-Cecil medicine. 26th ed. Philadelphia: Elsevier; 2020. p. 602-612.

2. Lu H, Stratton CW, Tang YW. Outbreak of pneumonia of unknown etiology in Wuhan, China: The mystery and the miracle. J Med Virol. 2020;92(4):401-402.

3. Hui DS, I Azhar E, Madani TA, Ntoumi F, Kock R, Dar O, et al. The continuing 2019-nCoV epidemic threat of novel coronaviruses to global health - The latest 2019 novel coronavirus outbreak in Wuhan, China. Int J Infect Dis. 2020;91:264-266.

4. Elhakim TS, Abdul HS, Pelaez Romero C, Rodriguez-Fuentes Y. Spontaneous pneumomediastinum, pneumothorax and subcutaneous emphysema in COVID-19 pneumonia: a rare case and literature review. BMJ Case Rep. 2020;13(12):e239489.

5. Sonia F, Kumar M. A complication of pneumothorax and pneumomediastinum in a non-intubated patient with COVID-19: a case report. Cureus. 2020;12(8):e10044.

6. Sihoe AD, Wong RH, Lee AT, Lau LS, Leung NY, Law KI, et al. Severe acute respiratory syndrome complicated by spontaneous pneumothorax. Chest. 2004;125(6):2345-2351.

7. Das KM, Lee EY, Al Jawder SE, Enani MA, Singh R, Skakni L, et al. Acute middle east respiratory syndrome coronavirus: temporal lung changes observed on the chest radiographs of 55 patients. AJR Am J Roentgenol. 2015;205(3):W267-W274.

8. Zantah M, Dominguez Castillo E, Townsend R, Dikengil F, Criner GJ. Pneumothorax in COVID-19 disease- incidence and clinical characteristics. Respir Res. 2020;21(1):236.

9. Martinelli AW, Ingle T, Newman J, Nadeem I, Jackson K, Lane ND, et al. COVID-19 and pneumothorax: a multicentre retrospective case series. Eur Respir J. 2020;56(5):2002697.

10. Chen N, Zhou M, Dong X, Qu J, Gong F, Han Y, et al. Epidemiological and clinical characteristics of 99 cases of 2019 novel coronavirus pneumonia in Wuhan, China: a descriptive study. Lancet. 2020;395(10223):507-513.

11. Yang X, Yu Y, Xu J, Shu H, Xia J, Liu H, et al. Clinical course and outcomes of critically ill patients with SARS-CoV-2 pneumonia in Wuhan, China: a single-centered, retrospective, observational study. Lancet Respir Med. 2020;8(5):475-81.

12. Miró $O$, Llorens $P$, Jiménez $S$, Piñera $P$, Burillo-Putze G, Martín $A$, et al. Frequency, risk factors, clinical characteristics, and outcomes of spontaneous pneumothorax in patients with coronavirus disease 2019: a case-control, emergency medicine-based multicenter study. Chest. 2021;159(3):1241-1255.

13. Fox SE, Akmatbekov A, Harbert JL, Li G, Quincy Brown J, Vander Heide RS. Pulmonary and cardiac pathology in African American patients with COVID-19: an autopsy series from New Orleans. Lancet Respir Med. 2020;8(7):681-686.

14. Xu Z, Shi L, Wang Y, Zhang J, Huang L, Zhang $C$, et al. Pathological findings of COVID-19 associated with acute respiratory distress syndrome. Lancet Respir Med. 2020;8(4):420-422.

15. Janssen ML, van Manen MJG, Cretier SE, Braunstahl GJ. Pneumothorax in patients with prior or current COVID-19 pneumonia. Respir Med Case Rep. 2020;31:101187.

16. Sun R, Liu H, Wang X. Mediastinal emphysema, giant bulla, and pneumothorax developed during the course of COVID-19 pneumonia. Korean J Radiol. 2020;21(5):541-544.

17. Park SJ, Park JY, Jung J, Park SY. Clinical manifestations of spontaneous pneumomediastinum. Korean J Thorac Cardiovasc Surg. 2016;49(4):287-291.

18. Frenkel MA, Lyons LL. Spontaneous pneumomediastinum. An unusual cause of a sore throat. Postgrad Med. 1991;89(1):257-259.

19. López Vega JM, Parra Gordo ML, Diez Tascón A, Ossaba Vélez S. Pneumomediastinum and spontaneous pneumothorax as an extrapulmonary complication of COVID-19 disease. Emerg Radiol. 2020;27(6):727-730.

20. RECOVERY Collaborative Group, Horby P, Lim WS, Emberson JR, Mafham M, Bell JL, et al. Dexamethasone in Hospitalized Patients with Covid-19. N Engl J Med. 2021;384(8):693-704.

21. Wang W, Gao R, Zheng Y, Jiang L. COVID-19 with spontaneous pneumothorax, pneumomediastinum and subcutaneous emphysema. J Travel Med. 2020;27(5):taaa062.

22. Bernheim A, Mei X, Huang M, Yang $Y$, Fayad ZA, Zhang N, et al. Chest CT findings in coronavirus disease-19 (COVID-19): relationship to duration of infection. Radiology. 2020;295(3):200463.

23. Liu K, Zeng Y, Xie P, Ye X, Xu G, Liu J, et al. COVID-19 with cystic features on computed tomography: A case report. Medicine (Baltimore). 2020;99(18):e20175.

24. Shi H, Han X, Jiang N, Cao Y, Alwalid O, Gu J, et al. Radiological findings from 81 patients with COVID-19 pneumonia in Wuhan, China: a descriptive study. Lancet Infect Dis. 2020;20(4):425-434.

25. Chang SH, Chen D, Paone D, Geraci TC, Scheinerman J, Bizekis C, et al. Thoracic surgery outcomes for patients with Coronavirus Disease 2019. J Thorac Cardiovasc Surg. 2021:S0022-5223(21)00168-9.

26. Aguinagalde B, Aranda JL, Busca P, Martínez I, Royo I, Zabaleta J; Grupo de trabajo de la GPC para el Manejo de Pacientes con Neumotórax espontáneo. SECT Clinical practice guideline on the management of patients with spontaneous pneumothorax. Cir Esp (Engl Ed). 2018;96(1):3-11.

27. Hallifax R, Wrightson JM, Bibby A, Walker S, Stanton A, De Fonseca D, et al. Pleural services during the COVID-19 pandemic-revised. The British Thoracic Society. 2020. [Accessed Jan 18, 2021] Available in: www.brit-thoracic.org.uk/document-library/quality-improvement/ covid-19/pleural-services-during-covid-19-pandemic/

28. Cardillo G, Facciolo F, Giunti R, Gasparri R, Lopergolo M, Orsetti $\mathrm{R}$, et al. Videothoracoscopic treatment of primary spontaneous pneumothorax: a 6-year experience. Ann Thorac Surg. 2000;69(2):357361; discussion 361-362.

29. Aiolfi A, Biraghi T, Montisci A, Bonitta G, Micheletto G, Donatelli F, et al. Management of persistent pneumothorax with thoracoscopy and bleb resection in COVID-19 patients. Ann Thorac Surg. 2020;110(5):e413-e415.

30. Shan S, Guangming L, Wei L, Xuedong Y. Spontaneous pneumomediastinum, pneumothorax and subcutaneous emphysema in COVID-19: case report and literature review. Rev Inst Med Trop Sao Paulo. 2020;62:e76. 
31. Quincho-Lopez A, Quincho-Lopez DL, Hurtado-Medina FD. Case report: pneumothorax and pneumomediastinum as uncommon complications of COVID-19 pneumonia-literature review. Am J Trop Med Hyg. 2020;103(3):1170-1176.

32. Oye M, Ali A, Kandah F, Chowdhury N. Two cases of spontaneous pneumomediastinum with pneumothorax in patients with COVID-19 associated pneumonia. Respir Med Case Rep. 2020;31:101308.

33. Eperjesiova B, Hart E, Shokr M, Sinha P, Ferguson GT. Spontaneous Pneumomediastinum/Pneumothorax in Patients With COVID-19. Cureus. 2020;12(7):e8996.

34. Selvaraj V, Dapaah-Afriyie K. Iatrogenic pneumothorax and pneumomediastinum in a patient with COVID-19. R I Med J (2013). 2020;103(10):32-33.

35. Hazariwala V, Hadid H, Kirsch D, Big C. Spontaneous pneumomediastinum, pneumopericardium, pneumothorax and subcutaneous emphysema in patients with COVID-19 pneumonia, a case report. J Cardiothorac Surg. 2020;15(1):301.

36. Xiang C, Wu G. SARS-CoV-2 pneumonia with subcutaneous emphysema, mediastinal emphysema, and pneumothorax: a case report. Medicine (Baltimore). 2020;99(20):e20208.
37. Ucpinar BA, Sahin C, Yanc U. Spontaneous pneumothorax and subcutaneous emphysema in COVID-19 patient: case report. J Infect Public Health. 2020;13(6):887-889.

38. Tucker L, Patel S, Vatsis C, Poma A, Ammar A, Nasser W, et al. Pneumothorax and Pneumomediastinum Secondary to COVID-19 disease unrelated to mechanical ventilation. Case Rep Crit Care. 2020;2020:6655428.

39. Gillespie M, Dincher N, Fazio P, Okorji O, Finkle J, Can A. Coronavirus disease 2019 (COVID-19) complicated by spontaneous pneumomediastinum and pneumothorax. Respir Med Case Rep. 2020;31:101232.

40. Vela Colmenero RM, Pola Gallego de Guzmán MD, Molina de la Torre MC. Spontaneous pneumothorax and pneumomediastinum in bilateral pneumonia due to COVID-19. Med Intensiva. 2020;44(9):591-592.

Conflicto de intereses: Los autores declaran no tener conflicto de intereses. 